\title{
ON THE HOMEOMORPHISMS WHICH SATISFY THE POINCARÉ RECURRENCE THEOREM
}

\author{
CHUNG-WU $\mathrm{HO}^{1}$
}

\begin{abstract}
It is shown that for a large class of spaces, almost all the homeomorphisms of the space do not satisfy the Poincare Recurrence Theorem. More specifically, let $X$ be a compact manifold with a nonzero Euler characteristic and $H(X)$ be the space of all homeomorphisms of $X$ onto $X . H(X)$ is given the compact open topology. Then the set of all the homeomorphisms of $X$ which satisfy the Poincaré Recurrence Theorem is nowhere dense in $H(X)$.
\end{abstract}

Let $X$ be a Hausdorff space with a countable base and $\phi$ be a homeomorphism from $X$ onto itself. A point $x \in X$ is said to be recurrent under $\phi$ if $x$ is a limit point of the sequence $\left\{\phi^{n} x\right\}_{n=0}^{\infty}$. The Poincare Recurrence Theorem asserts that if $X$ has a finite Borel measure and $\phi$ preserves measure, then the set of points of $X$ which are not recurrent under $\phi$ is of measure zero [1], [8]. There have been attempts to extend the Poincare Recurrence Theorem to a wider class of homeomorphisms, for instance, E. Hopf studied the case where the measure on $X$ is not necessarily finite [6], [8] and recently, $\mathrm{R}$. Kurth studied the case where the homeomorphism $\phi$ is not necessarily measure preserving [7]. Instead of trying to find a direct extension of the theorem, we ask what kind of homeomorphism can satisfy the Poincaré Recurrence Theorem. In this note we shall show that for a large class of spaces, in a certain sense, almost all the homeomorphisms do not satisfy the Recurrence Theorem. To state the result more precisely, we introduce the following notations. We shall use $\mu$ to denote any given Borel measure on the space $X$ which assigns a positive value to each nonempty open subset of $X$ and use $H(X)$ to denote the space of all homeomorphisms of $X$ onto $X$. The space $H(X)$ will be given the compact open topology. An element $\phi \in H(X)$ will be called recurrent if the set of all nonrecurrent points of $\phi$ has measure zero with respect to $\mu$. Our result can now be stated in the following

THEOREM. For any closed manifold $X$ of $\operatorname{dim} \geqslant 1$ with a nonzero Euler characteristic, the set of all recurrent homeomorphisms of $X$ is nowhere dense in $H(X)$.

It is well known that on a closed manifold $X$, there always exists a Borel

Received by the editors October 20, 1975.

AMS (MOS) subject classifications (1970). Primary 54H20; Secondary 57E05, 34C35, 34D99.

Key words and phrases. Poincaré Recurrence Theorem, Borel measure, recurrent homeomorphism, Euler characteristic, nowhere dense subset.

${ }^{1}$ The author is grateful to Dr. Rudolf Kurth for introducing him to the field of Topological Dynamics.

(1) American Mathematical Society 1976 
measure which assigns a positive value to each nonempty open subset of $X$. Thus, the assumption on the existence of such measure does not impose any restriction on the manifold in our theorem. We do not know whether the theorem is true without the assumption that the Euler characteristic of $X$ is nonzero. Some of the preliminaries will be collected in the first section. The theorem itself will be proved in the second section.

I. Preliminaries. In the following, we shall let $X$ be a Hausdorff space with a countable base. We assume that a Borel measure $\mu$ is given on $X$ which assigns a positive value to each nonempty open subset of $X$. First, we observe that a nonrecurrent homeomorphism of $X$ is a homeomorphism $\phi$ of $X$ whose nonrecurrent points form a set of positive measure. For this, we need the following proposition, which was known to Carathéodory [1] and was also implicitly proved in [8, pp. 447-450]. However, the proof given here is somewhat simpler.

PROPOSITION 1. For any homeomorphism $\phi$ of $X$, the set of all nonrecurrent points of $\phi$ is measurable.

Proof. Let $\phi$ be given. For each subset $A$ of $X$, we define the subset $e(A)$, the escaping part of $A$ under $\phi$, to be the set $\left\{x \in A \mid \phi^{n}(x) \notin A\right.$ for each positive integer $n\}$. Let $\left\{U_{n}\right\}_{n=1}^{\infty}$ be a countable base for the topology of $X$. It is easy to see that if $N$ is the set of all nonrecurrent points of $X$, then $N=\cup_{n=1}^{\infty} e\left(U_{n}\right)$. It is then sufficient to show that for any open set $U$, the set $e(U)$ is measurable. But $e(U)=U-\cup_{n=1}^{\infty} \phi^{n}(U)$ and $\phi$ is a homeomorphism, the set $e(U)$ is clearly Borel measurable.

REMARK 1 . To show that a homeomorphism $\phi$ is nonrecurrent, it is sufficient to construct a nonempty open set $U$ of $X$ such that $\phi^{n}(U) \cap U=\varnothing$ for all $n \geqslant 1$, for then $U$ is a set of nonrecurrent points of a positive measure.

In the proof of our theorem, we shall use the following two propositions. Proposition 2 is a result of F. B. Fuller [2], also see [3] and [4].

PRoposition 2. Let $X$ be a compact metric ANR whose Euler characteristic, $\chi(X)$, is nonzero. Then for each homeomorphism $\phi$ of $X$ onto $X$, some iterate of $\phi$ has a fixed point.

REMARK 2. Let $\phi$ be a homeomorphism of such a space $X$ and $x$ be a fixed point of some iterate of $\phi$. Suppose $k$ is the least positive integer such that $\phi^{k}(x)=x$. Then observe that the points $x, \phi(x), \ldots, \phi^{k-1}(x)$ are distinct from each other and $\phi$ is periodic on this set.

Proposition 3. Let $(X, \rho)$ be a compact metric space, and $f: X \rightarrow X$ be a continuous function. For any finite sequence of positive numbers $t_{1}, t_{2}, \ldots, t_{k}$, there exists a positive number $\delta$ such that if $g: X \rightarrow X$ is continuous and satisfies the condition that $\rho(f(x), g(x))<\delta$ for all $x \in X$, then for each $i=1,2, \ldots$, $k, \rho\left(f^{i}(x), g^{i}(x)\right)<t_{i}$ for all $x \in X$.

Proof. Let $f: X \rightarrow X$ be given. It is not difficult to show that for each positive number $t$, there exist positive numbers $s_{1}$ and $s_{2}$ such that for any continuous $g: X \rightarrow X$ with $\rho(f(x), g(x))<s_{1}$ for all $x \in X$, and for any two points $x$ and $y$ of $X$ with $\rho(x, y)<s_{2}$, we have that $\rho(f(x), g(y))<t$.

We now prove the proposition by induction on $k$. The case for $k=1$ is 
trivial. Assuming the proposition to be true for $k-1$, we consider a sequence $t_{1}, t_{2}, \ldots, t_{k}$ of $k$ positive numbers. By the above observation, there exist positive numbers $s_{1}$ and $s_{2}$ for the number $t=t_{k}$. Then apply the induction hypothesis to the sequence $t_{1}, \ldots, t_{k-2}, \min \left(t_{k-1}, s_{2}\right)$, of $k-1$ positive numbers to produce a positive number $\delta_{1}$ such that for any continuous $g: X \rightarrow X$ with $\rho(f(x), g(x))<\delta_{1}$ for all $x \in X, \rho\left(f^{i}(x), g^{i}(x)\right)<t_{i}$ for $i$ $=1,2, \ldots, k-2$ and $\rho\left(f^{k-1}(x), g^{k-1}(x)\right)<\min \left(t_{k-1}, s_{2}\right)$. Now, let $\delta$ $=\min \left(\delta_{1}, s_{1}\right)$. The proposition is clearly satisfied with this $\delta$ for the sequence $t_{1}, t_{2}, \ldots, t_{k}$. This finishes the proof.

II. Proof of the theorem. In this section, we shall let $X$ be a closed $n(\geqslant 1)$ dimensional manifold with $\chi(X) \neq 0$. Let a Borel measure $\mu$ be given which assigns positive values on nonempty open subsets of $X$. Since we assume that all manifolds are Hausdorff and second countable, a metric $\rho$ can be chosen which induces the topology of $X[5, \mathrm{p}$. 75]. It is well known that the compact open topology on $H(X)$ is equivalent to the topology given by the sup-metric with respect to $\rho$. We shall denote the sup-metric on the space $H(X)$ again by $\rho$. Thus, to prove our theorem, it suffices to show that for any $\phi \in H(X)$ and any $\varepsilon$-neighborhood, $N(\phi, \varepsilon)$, of $\phi$ in $H(X)$, there exists an element $f \in N(\phi, \varepsilon)$ and a $\delta>0$ such that each element of the neighborhood $N(f, \delta)$ is nonrecurrent. (Thus, $N(f, \delta) \cap N(\phi, \varepsilon)$ forms a nonempty open subset of $N(\phi, \varepsilon)$ consisting of nonrecurrent homeomorphisms.)

Now, let $\phi \in H(X)$ and $\varepsilon>0$ be given. Since a compact manifold is always an ANR [9, p. 177], some iterate of $\phi$ must have a fixed point $x_{0}$ in $X$. By Remark 2, the orbit of $x_{0}$ is a set $\left\{x_{0}, \phi\left(x_{0}\right), \ldots, \phi^{k-1}\left(x_{0}\right)\right\}$ of $k$ distinct points, where $k$ is the least positive integer such that $\phi^{k}\left(x_{0}\right)=x_{0}$.

We now fix a neighborhood $U_{1}$ of $x_{0}$ so that $U_{1}, \phi\left(U_{1}\right), \ldots, \phi^{k-1}\left(U_{1}\right)$ are pairwise disjoint and diam $\left(\phi\left(U_{1}\right)\right)<\varepsilon$. Replacing $U_{1}$ by an open subset if necessary, we may assume that there is a coordinate function $h$ of the manifold $X$, defined on an open set containing the closure $\bar{U}_{1}$ of $U_{1}$ such that $h$ carries the point $x_{0}$ to the origin of $R^{n}$ and the set $U_{1}$ to the ball $B(0,1)$, where for each positive number $t, B(0, t)$ stands for the open ball of radius $t$ centered at the origin of $R^{n}$. We shall let $\overline{B(0, t)}$ denote the corresponding closed ball, and for each $t, 0<t \leqslant 1$, we shall let $U_{t}$ denote the set $h^{-1}(B(0, t))$ and $\bar{U}_{t}$ denote the set $h^{-1} \overline{(B(0, t))}$.

Now, fix a number $\tau<\frac{1}{3}$ such that $\phi^{k}\left(U_{\tau}\right) \subset U_{1 / 3}$. We may now construct an $f \in N(\phi, \varepsilon)$ as follows: First define a function $\eta: \overline{B(0,1)} \rightarrow \overline{B(0,1)}$ by

$$
\eta(x)= \begin{cases}\frac{3}{2} \tau x, & \text { if } 0 \leqslant|x| \leqslant \frac{2}{3}, \\ {\left[3(1-\tau)\left(|x|-\frac{2}{3}\right)+\tau\right] x,} & \text { if } \frac{2}{3} \leqslant|x| \leqslant 1\end{cases}
$$

where $|x|$ stands for the Euclidean norm of $x$. Observe that $\eta$ is a homeomorphism from $\overline{B(0,1)}$ onto $\overline{B(0,1)}$ which fixes the boundary of $\overline{B(0,1)}$ while it absorbs the ball $B\left(0, \frac{2}{3}\right)$ onto the ball $\overline{B(0, \tau)}$. Now define $f: X \rightarrow X$ by letting $f(x)=\phi(x)$ for $x \in X-U_{1}$ and $f(x)=\phi h^{-1} \eta h(x)$ for $x \in \bar{U}_{1}$. The map $f$ is clearly a homeomorphism of $X$ onto $X$. Also note that $f \in N(\phi, \varepsilon)$, for $f$ and $\phi$ differ only at points in the set $U_{1}$ and for each $x \in U_{1}$, the points $f(x)$ and $\phi(x)$ both belong to $\phi\left(U_{1}\right)$, hence, $\rho(f(x), \phi(x))<\operatorname{diam}\left(\phi\left(U_{1}\right)\right)<\varepsilon$. 
We shall now fix a $\delta>0$ so that each element of $N(f, \delta)$ is nonrecurrent. This is accomplished by a sequence of lemmas.

Lemma 1. For each integer $i=1,2, \ldots, k-1$,

$$
f^{i}\left(\bar{U}_{2 / 3}\right) \subset \phi^{i}\left(U_{1 / 3}\right) \text { and } f^{k}\left(\bar{U}_{2 / 3}\right) \subset U_{1 / 3}
$$

where $k$ is the least positive integer such that $\phi^{k}\left(x_{0}\right)=x_{0}$.

Proof. It suffices to prove that $f^{i}\left(\bar{U}_{2 / 3}\right)=\phi^{i}\left(\bar{U}_{\tau}\right)$ for each $i=1,2, \ldots, k$ since the lemma is clearly satisfied if we replace $f^{i}\left(\bar{U}_{2 / 3}\right)$ by $\phi^{i}\left(\bar{U}_{\tau}\right)$ for each $i$. For $i=1, f^{i}\left(\bar{U}_{2 / 3}\right)=\phi^{i}\left(\bar{U}_{\tau}\right)$ follows from the definition of $f$. Now for each $i, 1<i \leqslant k$, consider the set $f^{i}\left(\bar{U}_{2 / 3}\right)=f\left(f^{i-1}\left(\bar{U}_{2 / 3}\right)\right)$. We may use the induction hypothesis to write this set as $f\left(\phi^{i-1}\left(\bar{U}_{\tau}\right)\right)$. Since $\phi^{i-1}\left(\bar{U}_{\tau}\right)$ $\subset \phi^{i-1}\left(U_{1}\right)$ is disjoint from $U_{1}$ and $f$ agrees with $\phi$ outside $U_{1}$, we now have $f^{i}\left(\bar{U}_{2 / 3}\right)=f\left(\phi^{i-1}\left(\bar{U}_{\tau}\right)\right)=\phi\left(\phi^{i-1}\left(\bar{U}_{\tau}\right)\right)=\phi^{i}\left(\bar{U}_{\tau}\right)$. This proves Lemma 1.

LEMMA 2. There exists a $\delta>0$ such that for each $g \in N(f, \delta), g^{i}\left(\bar{U}_{2 / 3}\right)$ $\subset \phi^{i}\left(U_{1 / 3}\right)$ for each $i=1,2, \ldots, k-1$ and $g^{k}\left(\bar{U}_{2 / 3}\right) \subset U_{1 / 3}$.

Proof. For each $i=1,2, \ldots, k-1$, the preceding lemma shows that the compact set $f^{i}\left(\bar{U}_{2 / 3}\right)$ is contained in the open set $\phi^{i}\left(U_{1 / 3}\right)$ of $X$. Thus, the distance $\rho\left(f^{i}\left(\bar{U}_{2 / 3}\right), X-\phi^{i}\left(U_{1 / 3}\right)\right)$ is a positive number. Let $t_{i}$ be this number for each $i=1,2, \ldots, k-1$. Similarly, the number

$$
t_{k}=\rho\left(f^{k}\left(\bar{U}_{2 / 3}\right), X-U_{1 / 3}\right)
$$

is positive. Now apply Proposition 3 of $\$ 1$ to the function $f$ and the sequence of numbers $t_{1}, t_{2}, \ldots, t_{k}$, we immediately get a positive number $\delta$ with the desired properties.

We now choose a $\delta>0$ satisfying the conditions given by Lemma 2 . We need now show that each element of $N(f, \delta)$ is nonrecurrent. To do this, by Remark 1 of $\S 1$, we need only construct a nonempty open set $V$ such that for each positive integer $i, g^{i}(V) \cap V=\varnothing$ for each element $g \in N(f, \delta)$. This is done in the following lemma.

Lemma 3. Let $V$ be the open set $U_{2 / 3}-\bar{U}_{1 / 3}$. For any element $g \in N(f, \delta)$ and any positive integer $i, g^{i}(V) \cap V=\varnothing$.

Proof. Let an arbitrary element $g \in N(f, \delta)$ be given. We first show that for each positive integer $m, g^{m k}(V) \subset U_{1 / 3}$. The case for $m=1$ is a direct consequence of Lemma 2. The case for $m>1$ may then be proved easily by using Lemma 2 and applying induction on the integer $m$. Since $V$ is disjoint from $\bar{U}_{1 / 3}$, the inclusion $g^{m k}(V) \subset U_{1 / 3}$ implies in particular that $g^{m k}(V)$ $\cap V=\varnothing$ for each positive integer $m$. Now consider a positive integer $i$ which is not a multiple of $k$. Write $i=m k+r$ for some integer $m \geqslant 0$ and $r$ with 0 $<r<k$. Then $g^{i}(V)=g^{r}\left(g^{m k}(V)\right) \subset g^{r}\left(U_{2 / 3}\right)$. By Lemma 2 again, we see that $g^{i}(V) \subset \phi^{r}\left(U_{1 / 3}\right)$. But for $0<r<k, \phi^{r}\left(U_{1 / 3}\right) \subset \phi^{r}\left(U_{1}\right)$ is disjoint from $U_{1}$, hence, we again have $g^{i}(V) \cap V=\varnothing$. This proves Lemma 3 , and therefore, also the main theorem. 


\section{REFERENCES}

1. C. Carathéodory, Über den Wiederkehrsatz von Poincaré, Preuss. Akad. Wiss. 34 (1919), $580-584$.

2. F. B. Fuller, The existence of periodic points, Ann. of Math. (2) 57 (1953), 229-230. MR 14, 669.

3. O. Hájek, Homological fixed point theorems. I, II, III, Comment. Math. Univ. Carolinae 5 (1964), 13-31; 85-92; 6 (1965), 157-164. MR 31 \#1672; 33 \#3292.

4. B. Halpern, Fixed points for iterates, Pacific J. Math. 25 (1968), 255-275. MR 41 \# 1039.

5. J. G. Hocking and G. S. Young, Topology, Addison-Wesley, Reading, Mass., 1961. MR 23 \# A2857.

6. E. Hopf, Zwei Sätze über den wahrscheinlichen Verlauf dynamisher Systeme, Math. Ann. 103 (1930), 710-719.

7. R. Kurth, Some remarks on Poincaré's recurrence theorem, Preprints in Mathematics and Mathematical Sciences, no. 32, Southern Ill. Univ. at Edwardsville, 1975.

8. V. V. Nemyckiǐ and V. V. Stepanov, Qualitative theory of differential equations, OGIZ, Moscow, 1947; English transl., Princeton Math. Ser., no. 22, Princeton Univ. Press, Princeton, N. J., 1960. MR 10, 612; 22 \#12258.

9. H. Schubert, Topologie. Eine Einführung, Teubner, Stuttgart, 1964; English transl., Allyn and Bacon, Boston, Mass., 1968. MR 30 \#51; 37 \#2160.

Department of Mathematical Studies, Southern Illinois University at EdWardsville, EDWARDSVILLE, ILLINOIS 62025 